\title{
Letter to the editor: how the COVID-19 pandemic has changed outpatient diagnosis in the andrological setting
}

\author{
R. Mazzilli ${ }^{1}$ (D) V. Zamponi ${ }^{1} \cdot$ A. Faggiano $^{1}$
}

Received: 19 July 2021 / Accepted: 1 September 2021 / Published online: 10 September 2021

(C) Italian Society of Endocrinology (SIE) 2021

Keywords Coronavirus disease 2019-COVID-19 - Severe acute respiratory syndrome coronavirus 2-SARS-CoV-2 . Erectile dysfunction · Infertility $\cdot$ Hypogonadism

Dear Editor,

The Coronavirus disease 2019 (COVID-19), caused by the Severe acute respiratory syndrome coronavirus 2 (SARSCoV-2), has been declared a pandemic by the World Health Organization.

The pandemic is causing social and economic implications and is also suggested to influence childbearing intentions and to induce a decline in the birth rate [1]. Alongside reproductive status, sexual health is also being affected in the general population $[2,3]$. To this regard, a significant effect of COVID-19 on the development of erectile dysfunction (ED), independently of other variables (psychological status, age, and body mass index), has been described [2].

In light of these data, we describe the changes observed in the diagnostic circumstances of patients admitted at the andrological unit. For this purpose, the number and type of andrological consultation have been compared between the period "March-December 2020" and "March-December 2019". In case of more than one motivation, the patients were classified according to the most relevant condition judged by the patient himself.

Statistical analysis was carried out with GraphPadInStat software (Version 3.06 for Windows, San Diego, CA, USA). Fisher's exact test was used for analyzing data. A $p$ value $<0.05$ was considered statistically significant.

First, we observed a decline in the number of patients admitted for first andrological examinations, both considering the absolute number and the booking rates (586

R. Mazzilli

rossella.mazzilli@uniroma1.it; rossella.mazzilli@gmail.com

1 Department of Clinical and Molecular Medicine, University of Rome "Sapienza", Sant'Andrea Hospital, Via di Grottarossa 1038, Rome, Italy visits out of 650 slots available (90.2\%) in 2019 compared to $165 / 300(55.0 \%)$ in $2020 ; p=0.001)$. However, this is mainly explained by the restrictions imposed by the Italian government regarding outpatient's hospital services. In fact, the overall availability for consultation was decreased, as a consequence of lockdown policy and reduced healthcare personnel. Second, the prevalence of ED was significantly higher in 2020 compared to 2019 (86/165, 52.1\% vs 239/586, 40.8\%; $p=0.01$ ) (Table 1). Similarly, admission for low desire was significantly higher in 2020 compared to $2019(8 / 165,4.8 \%$ vs $10 / 586,1.7 \% ; p=0.04)$. Third, an andrological check-up for fertility as well as male partners of infertile couples was significantly reduced in 2020 compared to $2019(29 / 165,17.6 \%$ vs $137 / 586,23.4 \%$; $p=0.04)$.

No differences were observed between 2020 and 2019 as for pain, inflammation or infection of the genital tract $(12 / 165,7.3 \%$ vs $53 / 586,9.0 \% ; p=0.5)$, varicocele $(7 / 165$, $4.2 \%$ vs $29 / 586,4.9 \% ; p=0.6)$, induratio penis plastica $(9 / 165,5.4 \%$ vs $36 / 586,6.1 \% ; p=0.9)$, gynecomastia $(2 / 165$, $1.2 \%$ vs $5 / 586,0.8 \% ; p=0.7)$, testicular tumors $(1 / 165,0.6 \%$ vs $3 / 586,0.5 \% ; p=1.0)$, puberty status assessment $(1 / 165$, $0.6 \%$ vs $3 / 586,0.5 \% ; p=1.0)$, hypogonadism $(7 / 165,4.2 \%$ vs $30 / 586,5.1 \% ; p=0.8)$, and premature ejaculation $(3 / 165$, $1.8 \%$ vs $28 / 586,4.8 \%$; $p=0.1$ ). Furthermore, in 2020 , no admission for ejaculatory dysfunction, phimosis and gender identity/sexual orientation were done, as compared to $1.7 \%$, $0.3 \%$ and $0.2 \%$, respectively, in 2019 ).

During the first national lockdown, between March and May 2020, only 11 first visits were done. Considering the motivation, the prevalence of pain, inflammation or infection of the genital tract and hypogonadism was significantly higher compared to the following months (June-December 2020) $(5 / 11,45.5 \%$ vs $7 / 154,4.5 \%, p=0.003$, and $2 / 11$, $18.2 \%$ vs $5 / 154,3.2 \%, p=0.001$, respectively). On the other hand, the prevalence of ED was significantly reduced (2/11, 
Table 1 Diagnostic circumstances of first admission for andrological abnormalities in 2019 and in 2020

\begin{tabular}{llll}
\hline & $\begin{array}{l}2019 \\
\text { (total admitted 586) }\end{array}$ & $\begin{array}{l}2020 \\
\text { (total admitted 165) }\end{array}$ & $p$ value \\
\hline Erectile dysfunction & $239(40.8 \%)$ & $86(52.1 \%)$ & 0.01 \\
Low desire & $10(1.7 \%)$ & $8(4.8 \%)$ & 0.03 \\
Infertility & $137(23.4 \%)$ & $29(17.6 \%)$ & 0.04 \\
Pain, inflammation or infection & $53(9.0 \%)$ & $12(7.3 \%)$ & 0.53 \\
Varicocele & $29(4.9 \%)$ & $7(4.2 \%)$ & 0.8 \\
Induratio penis plastica & $36(6.1 \%)$ & $9(5.4 \%)$ & 0.9 \\
Gynecomastia & $5(0.8 \%)$ & $2(1.2 \%)$ & 0.7 \\
Testicular tumors & $3(0.5 \%)$ & $1(0.6 \%)$ & 1.0 \\
Puberty status assessment & $3(0.5 \%)$ & $1(0.6 \%)$ & 1.0 \\
Hypogonadism & $30(5.1 \%)$ & $7(4.2 \%)$ & 0.8 \\
Premature ejaculation & $28(4.8 \%)$ & $3(1.8 \%)$ & 0.1 \\
Phimosis & $10(1.7 \%)$ & - & $\mathrm{NE}$ \\
Ejaculatory dysfunction & $2(0.3 \%)$ & - & $\mathrm{NE}$ \\
Gender identity/sexual orientation & $1(0.2 \%)$ & - & $\mathrm{NE}$ \\
\hline
\end{tabular}

$N E$ not evaluable
$18.2 \%$ vs $84 / 154,54.5 \%, p=0.03)$. No differences were observed comparing fertility status assessment and induratio penis plastica $(1 / 11(9.0 \%)$ vs $28 / 154(18.2 \%)$, and $1 / 11$ $(9.0 \%)$ vs $8 / 154,5.2 \%$, respectively; $p=0.7)$.

Finally, in 2020, a total of 50 follow-up visits on 120 slots available (41.6\%), were performed. Even if the follow-up visits were also regulated by medical personnel judgment, we observed a similar trend of the motivations (ED: 23/50 cases, $46.0 \%$; fertility status assessment: $8 / 50,16.0 \%$; hypogonadism: 6/50, 12.0\%; pain, inflammation or infection of the genital tract: $5 / 50,10.0 \%$; varicocele: $4 / 50,8.0 \%$; induratio penis plastic: $4 / 50,8.0 \%$ ).

In conclusion, these data highlight a dramatic impact of the pandemic on sexual function, especially on erectile function, often perceived by the patients as something requiring immediate attention. To this regard, the increased psychological distress resulting mainly after lockdown period, on top of the pathophysiological mechanisms in COVID-19 affected individuals, represents one of the main drivers of sexual dysfunctions in the "general" population. On the other hand, in our experience of an Andrology unit in Italy, in 2020, male population could have underestimated the pathological disorders causing reproductive dysfunction, perceived as "less urgent" reasons, and such other severe andrological abnormalities, postponing controls and screening. Nevertheless, we cannot exclude whether the reduced request for infertility consultation could be a consequence of the reduced interest for sexual activity.
Availability of data and material Available.

Code availability Not applicable.

\section{Declarations}

Conflict of interest Authors' have no conflict of interest and this work was not supported by any external funding.

Ethical approval Not applicable.

Informed consent Not applicable.

Consent for publication Not applicable.

\section{References}

1. Aassve A, Cavalli N, Mencarini L, Plach S, Livi Bacci M (2020) The COVID-19 pandemic and human fertility. Science 369:370 371. https://doi.org/10.1126/science.abc9520 (PMID: 32703862)

2. Sansone A, Mollaioli D, Ciocca G, Colonnello E, Limoncin E, Balercia G, Jannini EA (2021) "Mask up to keep it up": preliminary evidence of the association between erectile dysfunction and COVID-19. Andrology. https://doi.org/10.1111/andr.13003 (Epub ahead of print)

3. Bulut EC, Ertaş K, Bulut D, Koparal MY, Çetin S (2021) The effect of COVID-19 epidemic on the sexual function of healthcare professionals. Andrologia 53:e13971. https://doi.org/10.1111/and. 13971

Publisher's Note Springer Nature remains neutral with regard to jurisdictional claims in published maps and institutional affiliations.

Funding Not applicable. 